\title{
Direct Observation of Oxygen Superstructures in Manganites
}

\author{
S. Grenier, ${ }^{1}$ K. J. Thomas, ${ }^{2}$ J. P. Hill, ${ }^{2}$ U. Staub, ${ }^{3}$ Y. Bodenthin, ${ }^{3}$ M. García-Fernández, ${ }^{3}$ V. Scagnoli, ${ }^{4}$ V. Kiryukhin, ${ }^{5}$ \\ S-W. Cheong, ${ }^{5}$ B. G. Kim, ${ }^{5, *}$ and J. M. Tonnerre ${ }^{1}$ \\ ${ }^{1}$ Institut Néel, CNRS \& Université Joseph Fourier, BP 166, F-38042 Grenoble Cedex 9, France \\ ${ }^{2}$ Condensed Matter Physics and Materials Science Department, Brookhaven National Laboratory, Upton, New York 11973, USA \\ ${ }^{3}$ Swiss Light Source, Paul Sherrer Institut, 5232 Villigen, Switzerland \\ ${ }^{4}$ European Synchrotron Radiation Facility, BP 220, F-38043 Grenoble Cedex 9, France \\ ${ }^{5}$ Department of Physics and Astronomy, Rutgers University, Piscataway, New Jersey 08854 USA
}

(Received 28 May 2007; published 15 November 2007)

\begin{abstract}
We report the observation of superstructures associated with the oxygen $2 p$ states in two prototypical manganites using $x$-ray diffraction at the oxygen $K$ edge. In the stripe order system $\mathrm{Bi}_{0.31} \mathrm{Ca}_{0.69} \mathrm{MnO}_{3}$, hole-doped $\mathrm{O}$ states are orbitally ordered, at the same propagation vector as the $\mathrm{Mn}$ orbital ordering, but no oxygen charge stripes are found at this periodicity. In $\mathrm{La}_{7 / 8} \mathrm{Sr}_{1 / 8} \mathrm{MnO}_{3}$, we observe a $2 p$ charge ordering described by alternating hole-poor and hole-rich $\mathrm{MnO}$ planes that is consistent with some of the recent predictions.
\end{abstract}

DOI: 10.1103/PhysRevLett.99.206403

PACS numbers: 71.30.+h, 61.10.Nz, 71.27.+a

Fascinating macroscopic properties may emerge from the electronic and magnetic orderings in doped $3 d$ metal oxides. In cuprates, charge and spin stripes could be relevant to high- $T_{C}$ superconductivity [1], whereas the colossal magnetoresistance in manganites is fundamentally related to the stability of the correlated orderings of polarons, charges, orbitals, and spins [2]. The relationship between the orderings and the transport properties has been addressed both theoretically and experimentally focusing on the $3 d$ metal atoms, often neglecting the ligands' degrees of freedom. However, the active states are not ionic $3 d$ metal orbitals, but rather hybridized metal $3 d$-oxygen $2 p$ states; therefore, an ordering of the oxygen must equivalently accompany an ordering of the metals. In cuprates, the role of the oxygen atom is now always considered, and, taking a recent example, the ordering of hole-doped oxygens was directly observed and discussed in the context of the stripes [3].

In manganites, an electronic ordering on oxygens has not been detected yet, although recent experiments have suggested a crucial role of the $\mathrm{O}$ atoms in the correlated orderings on $\mathrm{Mn}$ atoms. X-ray spectroscopy of $\mathrm{Mn}$ atoms has revealed a small charge disproportionation, emphasizing the inadequacy of the traditional model of strongly localized charge ordering $\left(\mathrm{Mn}^{4+} / \mathrm{Mn}^{3+}\right)$ [4]. The conductivity was also found related to the oxygen electronic configuration [5], and it was proposed that the oxygen atom bridges an electron between two Mn atoms of nearly the same valence, coupling them magnetically [6]. Actually, these results point out the role of the $2 p-3 d$ hybridization in determining the magnetic exchange couplings and conductivity, the traditional picture being the one where the atomic $3 d$ orbitals define exchange magnetic pathways between Mn atoms, the oxygen $2 p$ states being filled.

Meanwhile, new theoretical models beyond the Mncentered description also suggested that oxygen superstructures play an important role in determining the ground states. Ferrari et al. [7] explained the insulating behavior of the charge or orbitally ordered half-doped system by an ordering of $\mathrm{O}$ holes - "oxygen stripes" of varying valences (incidentally, having the same periodicity as the Mn orbital ordering). Efremov et al. [8] proposed that the charge ordering could correspond to a charge density wave $(\mathrm{CDW})$ that is either Mn centered or $\mathrm{O}$ centered, and that an intermediate CDW (an intermediate O-Mn charge transfer) would potentially give both ferromagnetism and ferroelectricity. Also, Volja et al. [9] insisted on the relevance of the Mn charge ordering concept, if the hybridization with the hole-doped $\mathrm{O}$ atoms is properly considered.

Experiments that directly probe the oxygen contribution to the ordered states provide essential feedback to these theoretical models. In this Letter, we show that $\mathrm{O} K$ edge resonant diffraction spectra reveal the oxygen superstructures in manganites. We first report the direct observation of the orbital ordering of hole-doped, hybridized, $\mathrm{O} 2 p$ states in a Mn-centered, Mn-orbitally ordered manganite, $\mathrm{Bi}_{1-x} \mathrm{Ca}_{x} \mathrm{MnO}_{3}(x=0.69)$. Second, we report the charge ordering on the oxygen in $\mathrm{La}_{7 / 8} \mathrm{Sr}_{1 / 8} \mathrm{MnO}_{3}$; our observations confirm the stacking of alternate hole-rich and holepoor planes. In both cases, the $\mathrm{O}$ states are directly related to the Mn states, emphasizing the hybridized nature of the active states involved in the electronic orderings.

$\mathrm{Bi}_{0.31} \mathrm{Ca}_{0.69} \mathrm{MnO}_{3}$ (BCMO) is representative of the $\mathrm{Mn}$ centered orbitally ordered CDW phase, which is usually described as Mn stripe order. This phase is robust in manganites, from half-doping to $x=0.8$ [2]. The CDW exhibits polaronic sites $\left(\mathrm{Mn}^{3+}\right)$ placed as far as possible from each other, with an antiferro order of the valence orbital, while $\mathrm{Mn}^{4+}$ sites are isotropic [10] (Fig. 1). As the doping increases, the polaron density diminishes, and a larger orbital ordering periodicity is obtained $(\Lambda \approx 17 \AA$ for $\mathrm{BCMO}$ ), which is large enough to diffract soft $\mathrm{x}$ rays near the $\mathrm{O} K$ edge $(\lambda=23.4 \AA$ at $530 \mathrm{eV})$. BCMO orders 

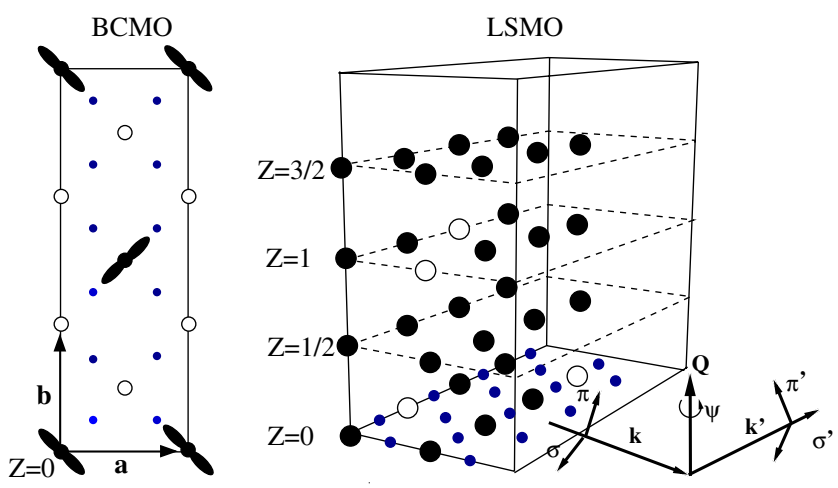

FIG. 1 (color online). Simplified orderings in BCMO and LSMO. Closed circles represents polaronic $\mathrm{Mn}^{3+}$ atoms, open circles are $\mathrm{Mn}^{4+}$, and small circles are the $\mathrm{O}$ sites; the orbital ordering in BCMO is also shown. The structure in the $a-b$ plane of BCMO repeats in adjacent planes, while LSMO planes alternate between being hole rich $(z=0$ and 1$)$ and hole poor $(z=$ $1 / 2$ and 3/2) [11]; for clarity the oxygens are represented in the first plane only. The scattering geometry is indicated, $\psi$ is the azimuthal angle, and $\sigma$ and $\pi$ are the two polarization components.

below $T_{\mathrm{OO}}=250 \mathrm{~K}$. The second system, $\mathrm{La}_{7 / 8} \mathrm{Sr}_{1 / 8} \mathrm{MnO}_{3}$ (LSMO), exhibits several phase transitions upon cooling. At $T_{\mathrm{OO} 1} \approx 280 \mathrm{~K}$, a polaronic insulating orbital ordering takes place. Then, the system becomes a metallic ferromagnet at $T_{C} \approx 180 \mathrm{~K}$ but finally stabilizes below $T_{\mathrm{OO} 2} \approx$ $150 \mathrm{~K}$ as an insulating ferromagnet. It was recently demonstrated that the $\mathrm{OO} 1$ to $\mathrm{OO} 2$ transition corresponds to a rearrangement of the polarons in a larger unit cell [11]. Here, we directly probed the electronic ordering that accompanies the polaron rearrangement allowing the test of two recent models for the electronic structures (described below).

The BCMO compound was prepared by the flux technique and LSMO by the floating zone technique; both samples were characterized by hard x-ray diffraction. The crystals were cut and polished with the normal to the surface along [010] for BCMO and along [001] for LSMO. We refer to the Pbnm orthorhombic notations for the unit cell.

The data were collected at the SIM beam line of the Swiss Light Source using the RESOXS setup built for soft $\mathrm{x}$-ray diffraction and reflectivity in ultrahigh vacuum [12]. The experiments were performed as in conventional diffraction but with the incident energy varied through the $\mathrm{O}$ $K$ edge while moving the diffractometer angles to maintain the diffraction condition. The $\mathrm{O} K$ edge corresponds to the photoelectric transition of a $1 \mathrm{~s}$ electron to unoccupied orbitals $\psi_{n}$. This transition is accounted for in the scattering factor by the overlap integral $\hat{\varepsilon} \cdot \int \psi_{n}^{*}(\mathbf{r}) \mathbf{r} \psi_{1 s}^{\mathrm{O}}(\mathbf{r}) d \mathbf{r}, \hat{\varepsilon}$ being the photon polarization. The resonant and the Thomson scattering factors are then weighted by the crystallographic phase factor at the selected Bragg reflection, $F(Q, \omega)=\sum_{j} f_{j}(Q, \omega) e^{i \mathbf{Q} \cdot \mathbf{r}_{j}}, \omega$ being the energy of the photon and the energy between $\psi_{n}$ and $\psi_{1 s}^{\mathrm{O}}$ [13]. By sweeping the incident photon energy through the $\mathrm{O} K$ edge, a resonant reflection provides evidence of the periodicity of unoccupied states $\psi_{n}$ from and above the Fermi level $\left(\varepsilon_{f}\right)$. The states probed are those without an even symmetry relative to the oxygen $1 s$ orbital $\psi_{1 s}^{\mathrm{O}}$ (which is even), as easily seen from the overlap integral. Near $\varepsilon_{f}$, those may be $\mathrm{O} 2 p$ 's, or orbitals from nearby atoms with some projection on the oxygens (e.g., $3 d$ ), or orbitals made of a hybridization of the two. Farther above $\varepsilon_{f}$, the states are more delocalized and may not carry an information on the electronic structure but rather on all surrounding atoms, i.e., a structural information. We also measured azimuthal scans (record of the reflection intensity while rotating the crystal in a plane normal to $\mathbf{Q}$ ), which allow one to rotate the polarization of the photon relatively to the atoms thereby probing different directions of the unoccupied states $\left(\psi_{2 p_{x}}, \ldots\right)$. We could select the two incident polarizations, namely $\sigma$ or $\pi$, perpendicular to and within the scattering plane, respectively (Fig. 1), the detector integrating photons of both polarizations.

Figure 2(a) shows the $\mathrm{O} K$ edge resonant $\mathrm{X}$-ray diffraction (RXD) spectrum at the $\left(\begin{array}{lll}0 & 0.31 & 0\end{array}\right)$ superstructure reflection we found in BCMO, for $\pi$ and $\sigma$ incident polarizations. No scattering is observed outside a narrow energy range $(1.1 \mathrm{eV})$ at the edge of the absorption spectrum (given by the fluorescence yield). We observed equal spectra for the two incident polarizations. As has been discussed elsewhere [10], this implies that the scattering process is one in which the polarization of the photon is rotated, of the type $\sigma \rightarrow \pi$ or $\pi \rightarrow \sigma$, that is, with zero $\sigma \rightarrow \sigma$ and $\pi \rightarrow \pi$ scattering. The incommensurability of this reflection is observed to vary with temperature, stabilizing at about 0.31 r.l.u., below $T_{\mathrm{OO}}$ [Fig. 2(b)]. A final observation, from Fig. 2(c), is the extinction of the reflection when the polarization of the incident photon is perpendicular to the ordered $(a, b)$ plane.

The first observation indicates a resonant superstructure reflection from an ordering of the very first unoccupied states from $\varepsilon_{f}$ projected on the $\mathrm{O}$ atoms $\left(\psi_{1 s}^{\mathrm{O}}\right)$, with the propagation $Q=0.31$ r.l.u., corresponding to the periodicity of the Mn orbital ordering [10]. Following earlier analysis of the absorption data [14], the comparison with the fluorescence yield allows us to identify the ordered states as the $2 p$ states hybridized with the $3 d$. The narrow spectral width observed reflects the width of the (unoccupied) hybridized band. The second observation demonstrates that there is no charge ordering on $\mathrm{O}$ atoms at this wave vector. Indeed, a CDW of this periodicity would scatter in the $\sigma \rightarrow \sigma$ channels, which is absent on this reflection to within the uncertainty of our measurements. Furthermore, a charge order scatters at all azimuthal angles $[15,16]$, at odds with the data. These three observations prove that the $\mathrm{O} 2 p$ states are hole doped and show orbital ordering, and not charge ordering at this propagation vec- 

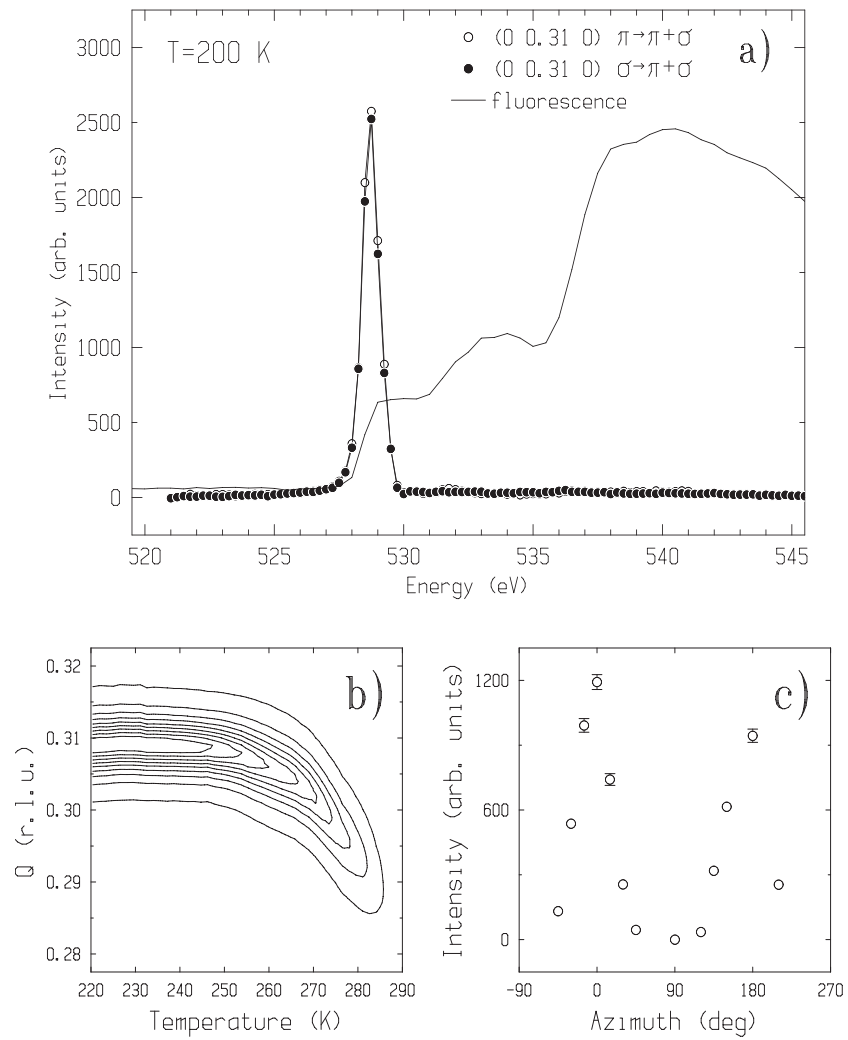

FIG. 2. (a) The $\left(\begin{array}{lll}0 & 0.31 & 0\end{array}\right)$ superstructure reflection, corrected from fluorescence but not for self-absorption; nevertheless, the intensity is zero off the $\mathrm{O} K$ edge. (b) Temperature dependence of the peak position at the phase transition. (c) Azimuthal dependence displaying a twofold symmetry with a maximum with the incident polarization along $\mathbf{a}$, and zero along $\mathbf{c}$, taken at $528.75 \mathrm{eV}, T=200 \mathrm{~K}$.

tor. Therefore, the data exclude a direct extension of the model of oxygen stripes, with the periodicity of the Mn orbital ordering, from half-doping [7] to this high doping.

The observation of $2 p$ orbital ordering makes intuitive sense in light of the $2 p-3 d$ hybridization and the earlier observation of this same orbital ordering, but projected on the Mn atoms $\left(\psi_{2 p}\right)$, at the Mn $2 p \leftrightarrow 3 d L$ edge [10]. Specifically, these states are centered on the polaronic Mn atoms, with a configuration depicted in Refs. $[9,17]$ clearly exhibiting the unoccupied $2 p$ ordering on the four $\mathrm{O}$ surrounding the polaronic $\mathrm{Mn}$. In agreement with this model, our results demonstrate the charge transfer nature of the compound and the coupling between the $\mathrm{O}$ and $\mathrm{Mn}$ orbital ordering.

We now turn to $\mathrm{La}_{7 / 8} \mathrm{Sr}_{1 / 8} \mathrm{MnO}_{3}$. Figure 3(a) shows the RXD spectra of the $\left(\begin{array}{lll}0 & 0 & \frac{1}{2}\end{array}\right)$ superstructure reflection at the $\mathrm{O} K$ edge for two incident polarizations. Previous studies have already reported the unusual stacking that doubles the unit cell along $\mathbf{c}$ below $T_{\mathrm{OO} 2}$ [18]; the present data also reveal a specific $2 p$-state reordering [Figs. 3(a) and 3(b)]. The fluorescence yields (proportional to $\operatorname{Im} f$ ) and the RXD spectra are different for the two polarizations; the differ-
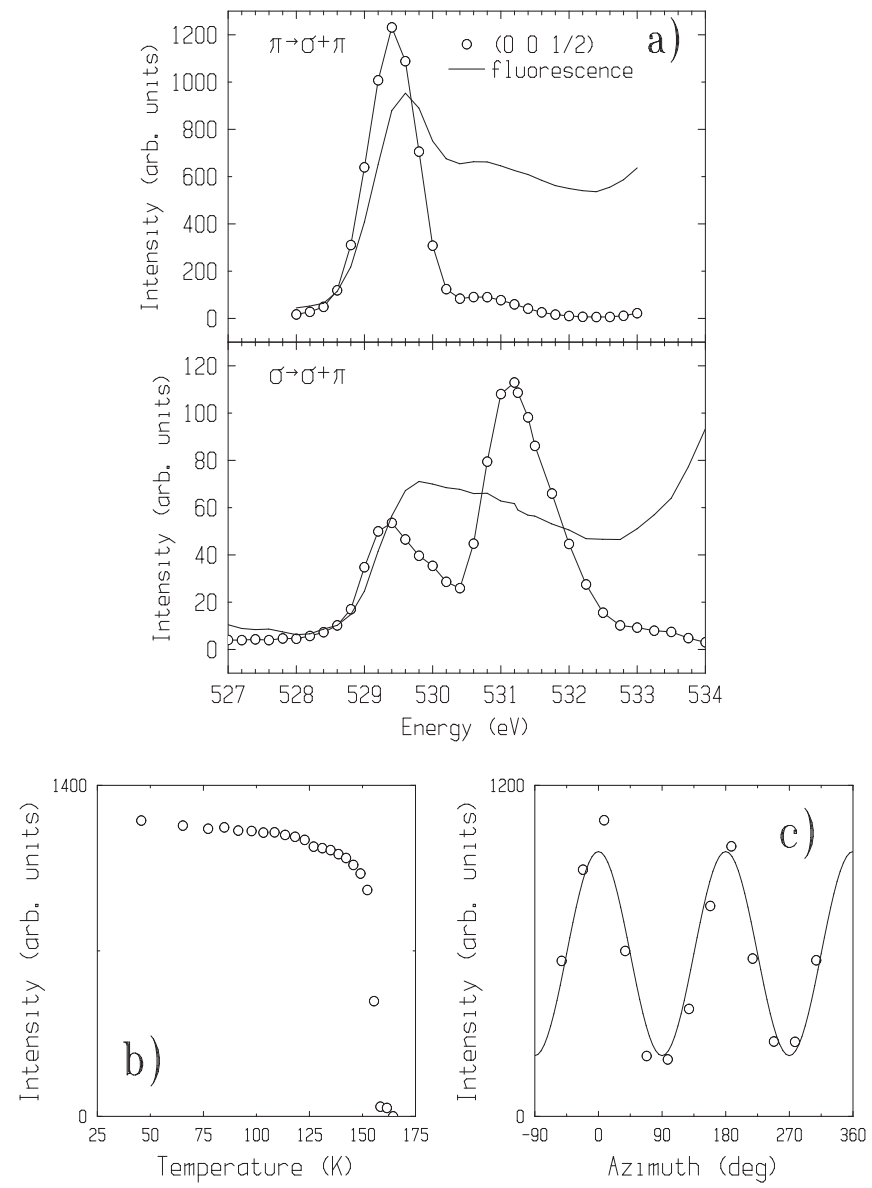

FIG. 3. (a) RXD spectra of the $\left(\begin{array}{lll}0 & 0 & \frac{1}{2}\end{array}\right)$ reflection at the $\mathrm{O} K$ edge of $\mathrm{La}_{7 / 8} \mathrm{Sr}_{1 / 8} \mathrm{MnO}_{3}$, with two incident polarizations, $T=$ $30 \mathrm{~K}, \psi \approx 90^{\circ}$. (b) Temperature dependence of the reflection. (c) Azimuthal dependence at $529.2 \mathrm{eV}, \sigma \rightarrow \sigma+\pi$ channels, compared to a $\cos ^{2}+$ const function (solid line), $0^{\circ}$ is $\sigma$ incident polarization along $\mathbf{b}, 90^{\circ}$ along $\mathbf{a}$.

ences provide evidence of an anisotropy of the unoccupied O states near $\varepsilon_{f}$. The main characteristic of the $2 p$ ordering is identified by considering the azimuthal dependence of the reflection. The azimuthal scan never goes to zero; it has a $\cos ^{2}+$ const dependence [Fig. 3(c)]. A tensorial analysis of the resonant scattering permits one to easily identify the origin of the two terms [16]: The constant term is due to a net difference in the total charge carried by the oxygens on different planes, whereas the sinusoidal dependence is due to the relative direction of the polarization of the photon with the anisotropic $\mathrm{O}$ electronic structure.

The diffraction vector $\left(\begin{array}{lll}0 & 0 & \frac{1}{2}\end{array}\right)$ corresponds to the net difference in scattering, hence to a net difference in holes, from planes separated by $\Delta z=1$; a charge ordering at the ( $\left(\begin{array}{lll}0 & 0 & \frac{1}{2}\end{array}\right)$ vector provides a strong constraint to the theoretical models for the charge ordering pattern. The models by Korotin et al. [19] and by Geck et al. [11] both propose that hole-rich and hole-poor Mn planes alternate along $\mathbf{c}$ but with different stacking orders. In Geck's model every other 


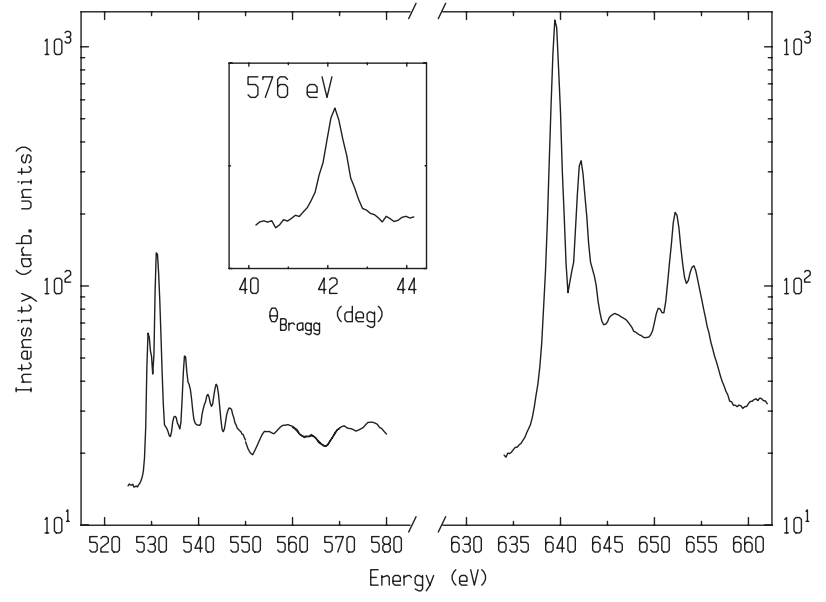

FIG. 4. RXD spectrum of the $\left(\begin{array}{lll}0 & 0 & \frac{1}{2}\end{array}\right)$ reflection of $\mathrm{La}_{7 / 8} \mathrm{Sr}_{1 / 8} \mathrm{MnO}_{3}, \sigma \rightarrow \sigma+\pi$ channels, $T=30 \mathrm{~K}$. The $\mathrm{O}$ near edge $(\approx 530 \mathrm{eV})$ and the $\mathrm{Mn} L_{\mathrm{III} / \mathrm{II}}$ edges $(\approx 640$ and $\approx 652 \mathrm{eV})$ probe the active states near $\varepsilon_{f}$, while the region between 535 and $560 \mathrm{eV}$ correspond to states that are delocalized (high energy from $\varepsilon_{f}$ ). Inset shows the diffraction peak at $576 \mathrm{eV}$, emphasizing the existence of the reflection far above the edge.

planes are identical when projected on the c axis: they should not give the charge order scattering we observe. In Korotin's model, three successive hole-rich planes alternate with one hole-poor plane. Such order should give rise to a strong charge order signal on the $\mathrm{O}$ atoms because of the hybridization, as we observe. We note that only Geck's model explains reflections with a planar component observed elsewhere [11].

Scattering is observed at this wave vector for energies well above the $\mathrm{O} K$ edge (Fig. 4). Increasing the energy from the near edge, the photoelectron probes states that are more delocalized, thus more sensitive to the crystal structure due to band structure effects. These features signal the presence of lattice distortions accommodating the charge ordering on oxygen atoms. At higher energy, the Mn $L$ edges correspond to the direct probe of the states nearby $\varepsilon_{f}$, like the O near edge, but probed by the Mn $2 p$ electrons and thus projected on the Mn atoms. Some of the near $\varepsilon_{f}$ hybridized states are probed at both edges, projected on $\mathrm{O}$ and $\mathrm{Mn}$, respectively. The intensities at both near edges in Fig. 4 provide the relative scattering strength of each atom, which should help determine the hybridization, charge transfer, and overlap between the $\mathrm{O}$ and $\mathrm{Mn}$ orbitals.

In conclusion, resonant $\mathrm{x}$-ray diffraction at the $\mathrm{O} K$ edge has been used to detect the electronic superstructures of the $2 p$ states near $\varepsilon_{f}$ in two manganites. In $\mathrm{Bi}_{0.31} \mathrm{Ca}_{0.69} \mathrm{MnO}_{3}$, we show evidence of an orbital ordering of hole-doped hybridized $2 p-3 d$, and contradict $\mathrm{O}$ charge stripes with the same propagation vector as the Mn orbital ordering. In $\mathrm{La}_{7 / 8} \mathrm{Sr}_{1 / 8} \mathrm{MnO}_{3}$, the data provided evidence of a charge ordering on the $\mathrm{O}$ atoms with the $\left(\begin{array}{lll}0 & 0 & \frac{1}{2}\end{array}\right)$ propagation vector, corresponding to a specific plane stacking with differing hole concentrations. The orderings on $\mathrm{O}$ atoms are not experimentally dissociated from the orderings on the Mn atoms, in both compounds, emphasizing the hybridized nature of the orbitals near $\varepsilon_{f}$. Quantification of the hole densities and of the $2 p-3 d$ hybridization requires $a b$ initio calculations of the ground state and the excited state using dedicated formalisms (the excited state has a strong Coulomb potential in a core shell and an extra electron in the valence shell). The RXD technique can also be chemically sensitive to the magnetic moment; therefore, these results suggest potential measurements of magnetic superstructures on the oxygens. Thus, the RXD data open a view on the $2 p-3 d$ states, the magnetic exchange couplings between $3 d$ metals and on the electronic superstructures at play in this strongly correlated system.

We benefited from the excellent support of the staff on X11MA-SLS, PSI. M. G.-F. thanks the Swiss National Science Foundation. This work was also supported by the U.S. Department of Energy, Division of Materials Science, under Contract No. DE-AC02-98CH10886. We thank J. Debray and S. Garaudée of the Institut Néel for excellent technical assistance.

*Present address: Department of Physics, Pusan National University, Pusan 609-735, Korea.

[1] J. M. Tranquada et al., Nature (London) 375, 561 (1995).

[2] Colossal Magnetoresistive Manganites, edited by T. Chatterji (Kluwer, Dordrecht, 2004).

[3] A. Rusydi et al., Phys. Rev. Lett. 97, 016403 (2006).

[4] G. Subías et al., Phys. Rev. B 56, 8183 (1997).

[5] M. Abbate et al., Phys. Rev. B 46, 4511 (1992).

[6] A. Daoud-Aladine et al., Phys. Rev. Lett. 89, 097205 (2002).

[7] V. Ferrari et al., Phys. Rev. Lett. 91, 227202 (2003).

[8] D. V. Efremov et al., Nature Mater. 3, 853 (2004).

[9] D. Volja et al., arXiv:0704.1834v1.

[10] S. Grenier et al., Phys. Rev. B 75, 085101 (2007).

[11] J. Geck et al., Phys. Rev. Lett. 95, 236401 (2005).

[12] N. Jaouen et al., J. Synchrotron Radiat. 11, 353 (2004).

[13] Resonant Anomalous X-Ray Scattering, Theory and Applications (Materlik, Sparks and Fisher, 1994).

[14] H. L. Ju et al., Phys. Rev. Lett. 79, 3230 (1997).

[15] J. García et al., J. Phys. Condens. Matter 13, 3243 (2001).

[16] S. Grenier et al., Phys. Rev. B 69, 134419 (2004).

[17] W.-G. Yin et al., Phys. Rev. Lett. 96, 116405 (2006).

[18] Y. Yamada et al., Phys. Rev. Lett. 77, 904 (1996).

[19] M. Korotin et al., Phys. Rev. B 62, 5696 (2000). 